\title{
APPROXIMATE CONTROLLABILITY OF IMPULSIVE RIEMANN-LIOUVILLE FRACTIONAL EQUATIONS IN BANACH SPACES
}

\author{
ZHENHAI LIU AND MAOJUN BIN
}

\begin{abstract}
In this paper, we study control systems governed by impulsive Riemann-Liouville fractional differential equations in Banach spaces. Firstly, we introduce $P C_{1-\alpha}$-mild solutions for impulsive Riemann-Liouville fractional differential equations. Then, we make a set of assumptions to guarantee the existence and uniqueness of mild solutions. Finally, approximate controllability of the associated impulsive Riemann-Liouville fractional evolution control systems is also formulated and proved.
\end{abstract}

1. Introduction. This paper is concerned with the approximate controllability of the following impulsive Riemann-Liouville fractional control systems:

(1.1)

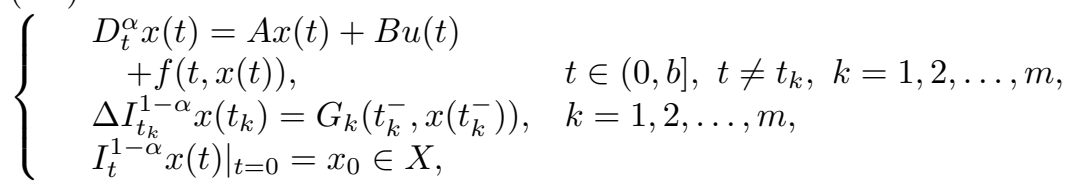

where $D_{t}^{\alpha}(0<\alpha \leq 1)$ denotes the Riemann-Liouville fractional derivative of order $\alpha$ with the lower limit zero. $A: D(A) \subseteq X \rightarrow X$ is the infinitesimal generator of a $C_{0}$-semigroup $T(t)(t \geq 0)$ on a Banach space $X . f, G_{k}: J \times X \rightarrow X$ are given functions to be specified later, where $J=[0, b] . \quad 0=t_{0}<t_{1}<\cdots<t_{m}<t_{m+1}=b$,

Keywords and phrases. Impulsive fractional evolution equations, RiemannLiouville fractional derivatives, $P C_{1-\alpha}$-mild solutions, approximate controllability.

Project supported by NNSF of China, Grant Nos. 11271087 and 61263006 and NSF of Guangxi, Grant No. 2014GXNSFDA118002. Also supported by the Marie Curie International Research Staff Exchange Scheme Fellowship within the 7th European Community Framework Programme under Grant Agreement No. 295118 and the National Science Center of Poland under Maestro Advanced Project No. UMO-2012/06/A/ST1/00262 and Special Funds of Guangxi Distinguished Experts Construction Engineering.

Received by the editors on June 30,2013, and in revised form on September 5, 2014. 
$\Delta I_{t_{k}}^{1-\alpha} x\left(t_{k}\right)=I_{t_{k}^{+}}^{1-\alpha} x\left(t_{k}^{+}\right)-I_{t_{k}^{-}}^{1-\alpha} x\left(t_{k}^{-}\right)=\Gamma(\alpha)\left[\lim _{t \rightarrow t_{k}^{+}}\left(t-t_{k}\right)^{1-\alpha} x(t)-\right.$ $\left.\lim _{t \rightarrow t_{k}^{-}}\left(t-t_{k}\right)^{1-\alpha} x(t)\right]$ ([14, Lemma 3.2, Chapter 3]). Furthermore, since $x\left(t_{k}^{-}\right)$is bounded (see the definition of $P C_{1-\alpha}(J, X)$ below), we can also write $\Delta I_{t_{k}}^{1-\alpha} x\left(t_{k}\right)=\Gamma(\alpha) \lim _{t \rightarrow t_{k}^{+}}\left(t-t_{k}\right)^{1-\alpha} x(t) . I_{t_{k}^{+}}^{1-\alpha} x\left(t_{k}^{+}\right)$ and $I_{t_{k}^{-}}^{1-\alpha} x\left(t_{k}^{-}\right)$denote the right and the left limits of $I_{t}^{1-\alpha} x(t)$ at $t=t_{k}, k=1,2, \ldots, m$. The control function $u$ takes its value in $V=L^{p}(J, U)(p>1 / \alpha)$, and $U$ is a Banach space. $B$ is a linear operator from $V$ into $L^{p}(J, X)$.

Since fractional differential equations provide an excellent instrument for the description of memory and hereditary properties in a model, they have drawn a great deal of applications in physics, aerodynamics, electrodynamics of complex medium, heat conduction, electricity mechanics and control theory. For more details on these topics, one can see for instance, $[\mathbf{2}, \mathbf{1 1}, \mathbf{1 4}, \mathbf{1 7}, \mathbf{2 8}, \mathbf{3 2}]$ and the references therein. The impulsive differential systems originate from the real world problems for describing the dynamic processes which are subjected to abrupt changes so that discontinuous jumps occur. Impulsive differential equations have become more and more important in various applications, such as control, physics, chemistry, population dynamics, aeronautics and engineering. For example, see $[7,19,20,23,25,26,27,38]$.

The concept of controllability, when it was first introduced by Kalman [13] in 1963, plays an important part in the analysis and design of control systems, and more details can be found in papers $[1,3,6,8,18]$. Some authors $[4,5,9]$ have studied the exact controllability for nonlinear evolution systems by using fixed point theorems. In these papers, in order to prove the controllability results for fractional order semilinear systems, the authors made the assumption that the corresponding linear operator associated with the $C_{0}$-semigroup $T(t)$ and the operator $B$ has a bounded inverse operator with values in the quotient space. But Triggiani [37] has pointed out that, if $C_{0}$-semigroup $T(t)$ is compact or the operator $B$ is compact, then the controllability operator is also compact. Hence, the inverse of the controllability operator would not exist if the state space $V$ is infinite dimensional. Thus, it is shown that the concept of exact controllability is difficult to be satisfied in infinite dimensional space. Therefore, it is important to study the weaker concept of controllability, namely, approximate controllability for differential equations. Recently, many 
researchers (cf., $[\mathbf{1 0}, \mathbf{1 5}, \mathbf{2 2}, \mathbf{2 7}, \mathbf{2 9}, \mathbf{3 3}, \mathbf{3 4}, \mathbf{3 5}, \mathbf{3 6}, \mathbf{4 0}])$ have studied it for different control systems.

On the other hand, Riemann-Liouville fractional derivatives or integrals are strong tools for resolving some fractional differential problems in the real world. It is possible to attribute physical meaning to initial conditions expressed in terms of Riemann-Liouville fractional derivatives or integrals which have been verified by Heymans and Podlubny [12], and such initial conditions are more appropriate than physically interpretable initial conditions. For another, they have considered the impulse response with Riemann-Liouville fractional derivatives as widely used in the fields of physics, such as viscoelasticity.

In recent years, many authors $[16,24,25,29,33,38,41]$ have investigated the existence and uniqueness of mild solutions and the controllability to fractional evolution equations with Caputo fractional derivative, and there have been a lot of interesting and excellent results on this fields. However, there is still little literature on the controllability of the fractional differential evolution with RiemannLiouville fractional derivatives. Very recently, Liu et al. [27] studied the existence and uniqueness of mild solutions and the approximate controllability for impulsive neutral evolution differential equations involving Riemann-Liouville fractional derivatives in Banach spaces. In order to prove the existence and uniqueness of mild solutions, the authors assumed that the following condition holds:

$$
L_{a g}\left\|A^{-1}\right\|+\frac{L_{a g} M b^{\alpha}}{\Gamma(\alpha+1)}+\sum_{i=1}^{k} M d_{i}\left(t-t_{i}\right)^{\alpha-1}+\frac{L_{f} M b^{\alpha}}{\Gamma(1+\alpha)}<1
$$

Unfortunately, this inequality is not true in general, by $\lim _{t \rightarrow t_{i}^{+}}(t-$ $\left.t_{i}\right)^{\alpha-1}=+\infty$ for $t \in\left(t_{i}, t_{i+1}\right]$. On the other hand, if $L_{a g} \neq 0$, the operator $A^{-1}$ may be unbounded (see [27, Theorem 3.2] for more details). Thus, it is important and meaningful to remove this hypothesis, and this fact is the motivation of the present work. On another hand, Liu et al. [21] studied the existence and uniqueness of mild solutions and the approximate controllability for impulsive Riemann-Liouville fractional evolution differential inclusions. In order to prove the existence of mild solutions, the authors assumed that the following condition holds: 
$H(4)$ : there exist constants $d_{k}>0, k=1,2, \ldots, m$, with

$$
\frac{M}{\Gamma(\alpha)} \sum_{k=1}^{m} d_{k}<1
$$

such that

$$
\left\|I_{k}(x)-I_{k}(y)\right\| \leq d_{k}\left(t-t_{k}\right)^{1-\alpha}\|x-y\|_{X}, \quad \text { for all } x, y \in X .
$$

Unfortunately, this inequality has some mistakes, by $\Delta I_{t_{k}}^{1-\alpha} x\left(t_{k}\right)=$ $I_{t_{k}^{+}}^{1-\alpha} x\left(t_{k}^{+}\right)-I_{t_{k}^{-}}^{1-\alpha} x\left(t_{k}^{-}\right)=\Gamma(\alpha)\left[\lim _{t \rightarrow t_{k}^{+}}\left(t-t_{k}\right)^{1-\alpha} x(t)-\lim _{t \rightarrow t_{k}^{-}}(t-\right.$ $\left.\left.t_{k}\right)^{1-\alpha} x(t)\right]$. It is shown that $\Delta I_{t_{k}}^{1-\alpha} x\left(t_{k}\right)$ does not include the variable $t$. In our paper, by introducing an appropriate weighted norm on the Banach space $P C_{1-\alpha}(J, X)$, we can obtain the existence and uniqueness of mild solutions for problems (1.1) which do not need the above inequality under the case $g(t, x(t)) \equiv 0$. It is noted that one can easily derive the main results for impulsive Riemann-Liouville fractional evolution systems on the literature $[\mathbf{2 1}, \mathbf{2 7}]$ under much weaker assumptions by the methods developed in this paper.

The rest of this paper is organized as follows. In Section 2, we will present some preliminaries which will be used to prove our main results. In Section 3, some sufficient conditions are established to guarantee the existence and uniqueness of mild solutions of the system (1.1). In Section 4, we will study the approximate controllability for fractional impulsive evolution differential equations with RiemannLiouville fractional derivatives. Finally, we present an example to illustrate our main results.

2. Preliminaries. In this section, we introduce some basic definitions and preliminaries which are used throughout this paper. The norm of a Banach space $X$ will be denoted by $\|\cdot\|_{X}$. For the uniformly bounded $C_{0}$-semigroup $T(t)(t \geq 0)$, we set $M:=\sup _{t \in[0, \infty)}\|T(t)\|<$ $\infty$. Let $A C(J, X)$ be the space of functions $f$ which are absolutely continuous on $J, A C^{m}(J, X)=\left\{f: J \rightarrow X\right.$ and $\left.f^{(m-1)}(x) \in A C(J, X)\right\}$. Let $C(J, X)$ denote the Banach space of all $X$-value continuous functions from $J=[0, b]$ into $X$ with the norm $\|x\|_{C}=\sup _{t \in J}\|x(t)\|_{X}$. Let $C_{1-\alpha}(J, X)=\left\{x: t^{1-\alpha} x(t) \in C(J, X)\right\}$ with the norm $\|x\|_{C_{1-\alpha}}=$ $\sup \left\{t^{1-\alpha}\|x(t)\|_{X}: t \in J\right\}$. Obviously, the space $C_{1-\alpha}(J, X)$ is a Banach space. 
In order to define the mild solutions of problems (1.1), we also consider the Banach space $P C_{1-\alpha}(J, X)=\left\{x:\left(t-t_{k}\right)^{1-\alpha} x(t) \in\right.$ $C\left(\left(t_{k}, t_{k+1}\right], X\right)$ and $\lim _{t \rightarrow t_{k}+}\left(t-t_{k}\right)^{1-\alpha} x(t)$ exists, $\left.k=0,1,2, \ldots, m\right\}$, with the norm

$$
\|x\|_{P C_{1-\alpha}}=\max \left\{\sup _{t \in\left(t_{k}, t_{k+1}\right]}\left(t-t_{k}\right)^{1-\alpha}\|x(t)\|_{X}: k=0,1,2, \cdots, m\right\} .
$$

Firstly, let us recall the following basic definitions from fractional calculus (cf., $[\mathbf{1 4}, \mathbf{3 2}])$ :

Definition 2.1. The integral

$$
I_{t}^{\alpha} f(t)=\frac{1}{\Gamma(\alpha)} \int_{0}^{t}(t-s)^{\alpha-1} f(s) d s, \quad \alpha>0,
$$

is called the Riemann-Liouville fractional integral of order $\alpha$, where $\Gamma$ is the gamma function.

Definition 2.2. For a function $f(t)$ given in the interval $[0, \infty)$, the expression

$$
D_{t}^{\alpha} f(t)=\frac{1}{\Gamma(n-\alpha)}\left(\frac{d}{d t}\right)^{n} \int_{0}^{t}(t-s)^{n-\alpha-1} f(s) d s,
$$

where $n=[\alpha]+1,[\alpha]$ denotes the integer part of number $\alpha$ and is called the Riemann-Liouville fractional derivative of order $\alpha$.

In order to study the $P C_{1-\alpha}$-mild solutions of problem (1.1), we need:

Lemma 2.3. ([27]). Let $0<\alpha \leq 1$, and let $x_{1-\alpha}(t)=I_{t}^{1-\alpha} x(t)$ be the fractional integral of order $1-\alpha$. If $x \in P C_{1-\alpha}(J, X)$ and $x_{1-\alpha} \in A C(J, X)$, then we have the following equality

$$
\begin{aligned}
& I_{t}^{\alpha} D_{t}^{\alpha} x(t) \\
&=\left\{\begin{array}{cc}
x(t)-\left.x_{1-\alpha}(t)\right|_{t=0} \frac{t^{\alpha-1}}{\Gamma(\alpha)}, & t \in\left[0, t_{1}\right], \\
x(t)-\sum_{i=1}^{k} \frac{\Delta x_{1-\alpha}\left(t_{i}\right)}{\Gamma(\alpha)}\left(t-t_{i}\right)^{\alpha-1} & \\
-\left.x_{1-\alpha}(t)\right|_{t=0} \frac{t^{\alpha-1}}{\Gamma(\alpha)}, & t \in\left(t_{k}, t_{k+1}\right],
\end{array}\right.
\end{aligned}
$$


where $\Delta x_{1-\alpha}\left(t_{k}\right)=x_{1-\alpha}\left(t_{k}^{+}\right)-x_{1-\alpha}\left(t_{k}^{-}\right), k=1,2, \ldots, m$.

Now, denote the Laplace transform formula of $x$ as follows:

$$
\widehat{x}(\lambda)=\int_{0}^{\infty} e^{-\lambda t} x(t) d t,
$$

$\operatorname{Re} \lambda>\omega$, and $|x(t)| \leq c e^{\omega t}, c$ is a constant.

Lemma 2.4. Let $0<\alpha \leq 1$ and $h \in L^{p}(J, X)(p>1 / \alpha)$. If $x \in P C_{1-\alpha}(J, X), x_{1-\alpha} \in P C(J, X)$ and $x$ is a solution of the following problem

$$
\begin{cases}D_{t}^{\alpha} x(t)=A x(t)+h(t), & t \in(0, b], t \neq t_{k}, k=1,2, \ldots, m, \\ & I_{t_{k}}^{1-\alpha} x\left(t_{k}\right)=y_{k}, \\ \left.I_{t}^{1-\alpha} x(t)\right|_{t=0}=x_{0} \in X, & k=1,2, \ldots, m,\end{cases}
$$

then $x$ satisfies the following equation

$$
x(t)=\left\{\begin{array}{l}
t^{\alpha-1} T_{\alpha}(t) x_{0}+\int_{0}^{t}(t-s)^{\alpha-1} T_{\alpha}(t-s) h(s) d s, \quad t \in\left[0, t_{1}\right], \\
t^{\alpha-1} T_{\alpha}(t) x_{0}+\sum_{i=1}^{k} T_{\alpha}\left(t-t_{i}\right)\left(t-t_{i}\right)^{\alpha-1} y_{i} \\
\quad+\int_{0}^{t}(t-s)^{\alpha-1} T_{\alpha}(t-s) h(s) d s, \quad t \in\left(t_{k}, t_{k+1}\right], \quad k=1, \ldots, m,
\end{array}\right.
$$

where

$$
\begin{gathered}
T_{\alpha}(t)=\alpha \int_{0}^{\infty} \theta \xi_{\alpha}(\theta) T\left(t^{\alpha} \theta\right) d \theta, \\
\xi_{\alpha}(\theta)=\frac{1}{\alpha} \theta^{-1-1 / \alpha} \varpi_{\alpha}\left(\theta^{-1 / \alpha}\right), \\
\varpi_{\alpha}(\theta)=\frac{1}{\pi} \sum_{n=1}^{\infty}(-1)^{n-1} \theta^{-n \alpha-1} \frac{\Gamma(n \alpha+1)}{n !} \sin (n \pi \alpha), \quad \theta \in(0, \infty),
\end{gathered}
$$

$\xi_{\alpha}$ is a probability density function defined on $(0, \infty)$, that is

$$
\xi_{\alpha}(\theta) \geq 0, \quad \theta \in(0, \infty)
$$

and

$$
\int_{0}^{\infty} \xi_{\alpha}(\theta) d \theta=1
$$


Proof. It is clear that $x(\cdot)$ can be decomposed to $\Phi(\cdot)+\Psi(\cdot)$, where $\Phi$ is the continuous mild solution for the differential equation

$$
\left\{\begin{array}{l}
D_{t}^{\alpha} \Phi(t)=A \Phi(t)+h(t), \quad t \in(0, b] \\
\left.I_{t}^{1-\alpha} \Phi(t)\right|_{t=0}=x_{0} \in X
\end{array}\right.
$$

and $\Psi$ is the $P C_{1-\alpha}$-mild solution for the impulsive differential equation (2.7)

$$
\begin{cases}D_{t}^{\alpha} \Psi(t)=A \Psi(t), & t \in(0, b], t \neq t_{k}, k=1,2, \ldots, m \\ \left.\Delta I_{t}^{1-\alpha} \Psi(t)\right|_{t=t_{k}}=y_{k}, & k=1,2, \ldots, m \\ \left.I_{t}^{1-\alpha} \Psi(t)\right|_{t=0}=0 \in X . & \end{cases}
$$

Indeed, since $\Phi$ is continuous, then $\Phi\left(t_{k}^{+}\right)=\Phi\left(t_{k}^{-}\right), k=1,2, \ldots, m$. Clearly, by adding (2.6) together with (2.7), (2.4) follows. On the other hand, any solution of (2.4) can be decomposed to (2.6) and (2.7). Now, we shall show the lemma in the following.

Firstly, from Pan et al. [30, Lemma 4], we know the mild solution of (2.6) is given by

$$
\Phi(t)=t^{\alpha-1} T_{\alpha}(t) x_{0}+\int_{0}^{t}(t-s)^{\alpha-1} T_{\alpha}(t-s) h(s) d s .
$$

Now, we consider the $P C_{1-\alpha}$-mild solution of (2.7). For this, applying Riemann-Liouville fractional integral operator on both sides of the equation (2.7), by Lemma 2.3 , we get

$$
\Psi(t)= \begin{cases}1 / \Gamma(\alpha) \int_{0}^{t}(t-s)^{\alpha-1} A \Psi(s) d s, & t \in\left[0, t_{1}\right] \\ \sum_{i=1}^{k} \frac{y_{i}}{\Gamma(\alpha)}\left(t-t_{i}\right)^{\alpha-1} & \\ & +\frac{1}{\Gamma(\alpha)} \int_{0}^{t}(t-s)^{\alpha-1} A \Psi(s) d s, \quad t \in\left(t_{k}, t_{k+1}\right] \\ & k=1, \ldots, m\end{cases}
$$

Obviously, equation (2.8) can be rewritten as

$$
\Psi(t)=\sum_{i=1}^{m} \frac{y_{i}}{\Gamma(\alpha)}\left(t-t_{i}\right)^{\alpha-1} \chi_{i}(t)+\frac{1}{\Gamma(\alpha)} \int_{0}^{t}(t-s)^{\alpha-1} A \Psi(s) d s, \quad t \in J,
$$

where

$$
\chi_{i}(t)= \begin{cases}0, & t \in\left[0, t_{i}\right) \\ 1, & t \in\left[t_{i},+\infty\right)\end{cases}
$$


Let $\lambda>0$. Taking the Laplace transformation to the equation (2.9), we obtain

$$
\widehat{\Psi}(\lambda)=\sum_{i=1}^{k} \frac{y_{i} e^{-\lambda t_{i}}}{\lambda^{\alpha}}+\frac{1}{\lambda^{\alpha}} A \widehat{\Psi}(\lambda),
$$

i.e.,

$$
\widehat{\Psi}(\lambda)=\sum_{i=1}^{k}\left(\lambda^{\alpha} I-A\right)^{-1} y_{i} e^{-\lambda t_{i}} .
$$

Notice that the Laplace transform of $t^{\alpha-1} T_{\alpha}(t) y_{i}$ is $\left(\lambda^{\alpha} I-A\right)^{-1} y_{i}$ (cf., $[30$, Lemma 4]). Thus, one can obtain the mild solution of (2.7) as

$$
\Psi(t)=\sum_{i=1}^{m} \chi_{i}(t)\left(t-t_{i}\right)^{\alpha-1} T_{\alpha}\left(t-t_{i}\right) y_{i}
$$

By the above arguments, the $P C_{1-\alpha}$-mild solution of (2.4) is given by

$$
\begin{aligned}
x(t)= & t^{\alpha-1} T_{\alpha}(t) x_{0}+\sum_{i=1}^{m} \chi_{i}(t)\left(t-t_{i}\right)^{\alpha-1} T_{\alpha}\left(t-t_{i}\right) y_{i} \\
& +\int_{0}^{t}(t-s)^{\alpha-1} T_{\alpha}(t-s) h(s) d s .
\end{aligned}
$$

This completes the proof of Lemma 2.4.

According to Lemma 2.4, we give the following definition.

Definition 2.5. A function $x \in P C_{1-\alpha}(J, X)$ is called a mild solution of problem (1.1) if it satisfies the following fractional integral equation

$$
x(t)=\left\{\begin{array}{c}
t^{\alpha-1} T_{\alpha}(t) x_{0}+\int_{0}^{t}(t-s)^{\alpha-1} T_{\alpha}(t-s) \\
{[B u(s)+f(s, x(s))] d s, \quad t \in\left[0, t_{1}\right]} \\
t^{\alpha-1} T_{\alpha}(t) x_{0}+\sum_{i=1}^{k} T_{\alpha}\left(t-t_{i}\right)\left(t-t_{i}\right)^{\alpha-1} G_{i}\left(t_{i}^{-}, x\left(t_{i}^{-}\right)\right) \\
+\int_{0}^{t}(t-s)^{\alpha-1} T_{\alpha}(t-s)[B u(s)+f(s, x(s))] d s \\
t \in\left(t_{k}, t_{k+1}\right], \quad k=1, \ldots, m
\end{array}\right.
$$

Definition 2.6. Let $x(\cdot ; u)$ be a mild solution of system (1.1) corresponding to the control $u(\cdot) \in V$ and the initial value $x_{0} \in X$. The set 
$K_{b}(f)=\{x(b ; u): u(\cdot) \in V\}$ is called the reachable set of system (1.1) at terminal time $b$. If $\overline{K_{b}(f)}=X$, then system (1.1) is said to be approximate controllable on $J$.

From the work of the paper [41], we have the following result:

Lemma 2.7. If the $C_{0}$-semigroup $T(t)(t \geq 0)$ is uniformly bounded (i.e., $\left.\sup _{t \in[0, \infty)}\|T(t)\| \leq M<\infty\right)$, then the operator $T_{\alpha}(t)$ has the following properties:

(i) For any fixed $t \geq 0, T_{\alpha}(t)$ is a linear and bounded operator, i.e., for any $x \in X$,

$$
\left\|T_{\alpha}(t) x\right\| \leq \frac{M}{\Gamma(\alpha)}\|x\| .
$$

(ii) $T_{\alpha}(t)(t \geq 0)$ is strongly continuous.

3. Existence of mild solutions. This section is devoted to the study of existence and uniqueness results for a class of fractional impulsive evolution differential equations involving Riemann-Liouville fractional derivatives.

In the sequel, we will make the following hypotheses on the data of our problems:

$H(1)$ : The function $f(\cdot, x): J \rightarrow X$ is measurable for all $x \in X$ and $\|f(t, 0)\| \in L^{p}\left(J, R^{+}\right), p>1 / \alpha$ for almost every $t \in J$. Moreover, there exists a constant $L>0$ such that

$$
\|f(t, x)-f(t, y)\| \leq L\left(t-t_{k}\right)^{1-\alpha}\|x-y\|_{X},
$$

for a.e. $t \in\left(t_{k}, t_{k+1}\right](k=0,1, \ldots, m)$ and any $x, y \in X$.

$H(2)$ : There exist constants $0<d_{k}<\Gamma(\alpha) /\left[2 M \sum_{i=1}^{k}\left(t_{i}-t_{i-1}\right)^{\alpha-1}\right]$ $(k=1,2, \ldots, m+1)$ (where $\left.M:=\sup _{t \in[0, \infty)}\|T(t)\|<\infty\right)$ such that

$$
\left\|G_{k}\left(t_{k}^{-}, x\right)-G_{k}\left(t_{k}^{-}, y\right)\right\| \leq d_{k}\|x-y\|_{X}, \quad \text { for all } x, y \in X .
$$

Now, we are in a position to prove the main result of this section. 
Theorem 3.1. Assume that the hypotheses $H(1)-H(2)$ are satisfied. Then, for each given control function $u(\cdot) \in V$, the initial problem (1.1) has a unique mild solution on $P C_{1-\alpha}(J, X)$.

Proof. Define the operator $\digamma: P C_{1-\alpha}(J, X) \rightarrow P C_{1-\alpha}(J, X)$ by

$$
(\digamma x)(t)=\left\{\begin{array}{c}
t^{\alpha-1} T_{\alpha}(t) x_{0}+\int_{0}^{t}(t-s)^{\alpha-1} T_{\alpha}(t-s) \\
{[B u(s)+f(s, x(s))] d s, \quad t \in\left[0, t_{1}\right]} \\
t^{\alpha-1} T_{\alpha}(t) x_{0}+\sum_{i=1}^{k} T_{\alpha}\left(t-t_{i}\right)\left(t-t_{i}\right)^{\alpha-1} G_{i}\left(t_{i}^{-}, x\left(t_{i}^{-}\right)\right) \\
+\int_{0}^{t}(t-s)^{\alpha-1} T_{\alpha}(t-s)[B u(s)+f(s, x(s))] d s \\
t \in\left(t_{k}, t_{k+1}\right], k=1, \ldots, m .
\end{array}\right.
$$

Then the problem of finding mild solutions for problem (1.1) is reduced to finding the fixed point of $\digamma$. To prove this, we consider the operator $\digamma$ on the Banach space $P C_{1-\alpha}(J, X)$ with a weighted norm

$$
\|x\|_{r}=\max \left\{\sup _{t \in\left(t_{k}, t_{k+1}\right]}\left(t-t_{k}\right)^{1-\alpha}\|x(t)\|_{X} e^{-r t}: k=0,1, \ldots, m\right\},
$$

where $r=\max \left\{\left(2 \Gamma(\alpha) M L b^{1-\alpha} / \Gamma(\alpha)-2 M \sum_{i=1}^{k} d_{i}\left(t_{i}-t_{i-1}\right)^{\alpha-1}\right)^{1 / \alpha}\right.$ : $k=1, \ldots, m+1\}$. Now, set $B_{r}(R)=\left\{x \in P C_{1-\alpha}(J, X):\|x\|_{r} \leq R\right\}$, where $R=2 \omega$ and

$$
\begin{aligned}
\omega= & \frac{M}{\Gamma(\alpha)}\left\|x_{0}\right\|+\frac{M}{\Gamma(\alpha)} \sum_{i=1}^{m}\left\|G_{i}\left(t_{i}, 0\right)\right\| \\
& +\frac{M b^{1-1 / p}}{\Gamma(\alpha)}\left(\frac{p-1}{p \alpha-1}\right)^{1-1 / p}\left[\|f(\cdot, 0)\|_{L^{p}\left(J, R^{+}\right)}+\|B u\|_{L^{p}(J, X)}\right] .
\end{aligned}
$$

Next, for the sake of convenience, we subdivide the proof into two steps.

Step 1 . We shall prove that the operator $\digamma$ maps $B_{r}(R)$ into itself.

Notice that

$$
\begin{aligned}
\int_{0}^{t}(t-s)^{\alpha-1} e^{r s} d s & =-r^{-\alpha} \int_{0}^{t}[r(t-s)]^{\alpha-1} e^{-r(t-s)} e^{r t} d[r(t-s)] \\
& =r^{-\alpha} e^{r t} \int_{0}^{r t} z^{\alpha-1} e^{-z} d z \quad(r(t-s)=z) \\
& \leq r^{-\alpha} e^{r t} \Gamma(\alpha)
\end{aligned}
$$


If $t \in\left[0, t_{1}\right]$, then from formula (3.1), condition $H(1)$ and the Hölder inequality, we obtain

$$
\begin{aligned}
t^{1-\alpha}\|(\digamma x)(t)\|_{X} \leq & \left\|T_{\alpha}(t) x_{0}\right\|+t^{1-\alpha} \\
& \times \int_{0}^{t}(t-s)^{\alpha-1}\left\|T_{\alpha}(t-s)[f(s, x(s))+B u(s)]\right\| d s \\
\leq & \frac{M}{\Gamma(\alpha)}\left\|x_{0}\right\|+\frac{M t^{1-\alpha}}{\Gamma(\alpha)} \int_{0}^{t}(t-s)^{\alpha-1}[\|f(s, 0)\| \\
& \left.+L s^{1-\alpha}\|x(s)\|_{X}+\|B u(s)\|_{X}\right] d s \\
\leq & \frac{M}{\Gamma(\alpha)}\left\|x_{0}\right\|+\frac{M b^{1-\alpha}}{\Gamma(\alpha)} \int_{0}^{t}(t-s)^{\alpha-1}[\|f(s, 0)\| \\
& \left.+\|B u(s)\|_{X}\right] d s+\frac{M L b^{1-\alpha}\|x\|_{r}}{\Gamma(\alpha)} \int_{0}^{t}(t-s)^{\alpha-1} e^{r s} d s \\
\leq & \frac{M}{\Gamma(\alpha)}\left\|x_{0}\right\|_{+} \frac{M b^{1-1 / p}}{\Gamma(\alpha)}\left(\frac{p-1}{p \alpha-1}\right)^{1-1 / p} \\
& \times\left[\|f(\cdot, 0)\|_{L^{p}\left(J, R^{+}\right)}+\|B u\|_{L^{p}(J, X)}\right] \\
& +M L b^{1-\alpha} e^{r t} r^{-\alpha}\|x\|_{r} .
\end{aligned}
$$

Thus, we have

$$
\sup _{t \in\left[0, t_{1}\right]} t^{1-\alpha}\|(\digamma x)(t)\|_{X} e^{-r t} \leq \omega+M L b^{1-\alpha} r^{-\alpha}\|x\|_{r} \leq R .
$$

If $t \in\left(t_{k}, t_{k+1}\right](k=1, \ldots, m)$, from formula (3.1), the assumptions $H(1), H(2)$ and the Hölder inequality, we have

$$
\begin{aligned}
\left(t-t_{k}\right)^{1-\alpha}\|(\digamma x)(t)\|_{X} & \leq\left(t-t_{k}\right)^{1-\alpha} t^{\alpha-1}\left\|T_{\alpha}(t) x_{0}\right\|+\left(t-t_{k}\right)^{1-\alpha} \\
& \times\left\|\sum_{i=1}^{k} T_{\alpha}\left(t-t_{i}\right)\left(t-t_{i}\right)^{\alpha-1} G_{i}\left(t_{i}^{-}, x\left(t_{i}^{-}\right)\right)\right\| \\
& +\left(t-t_{k}\right)^{1-\alpha} \\
& \times \int_{0}^{t}(t-s)^{\alpha-1}\left\|T_{\alpha}(t-s)[f(s, x(s))+B u(s)]\right\| d s \\
\leq & \frac{M}{\Gamma(\alpha)}\left\|x_{0}\right\|
\end{aligned}
$$




$$
\begin{aligned}
& +\frac{M}{\Gamma(\alpha)} \sum_{i=1}^{k} d_{i}\left(t_{i}-t_{i-1}\right)^{\alpha-1}\left(t_{i}-t_{i-1}\right)^{1-\alpha}\left\|x\left(t_{i}^{-}\right)\right\|_{X} \\
& +\frac{M}{\Gamma(\alpha)} \sum_{i=1}^{k}\left\|G_{i}\left(t_{i}, 0\right)\right\|+\frac{M\left(t-t_{k}\right)^{1-\alpha}}{\Gamma(\alpha)} \\
& \times \int_{0}^{t}(t-s)^{\alpha-1}\left[\|f(s, 0)\|+L\left(s-t_{k}\right)^{1-\alpha}\|x(s)\|_{X}\right. \\
& \left.+\|B u(s)\|_{X}\right] d s \\
& \leq \frac{M}{\Gamma(\alpha)}\left\|x_{0}\right\|+\frac{M}{\Gamma(\alpha)} \sum_{i=1}^{k}\left\|G_{i}\left(t_{i}, 0\right)\right\| \\
& +\frac{M b^{1-1 / p}}{\Gamma(\alpha)}\left(\frac{p-1}{p \alpha-1}\right)^{1-1 / p} \\
& \quad \times\left[\|f(\cdot, 0)\|_{L^{p}\left(J, R^{+}\right)}\|B u\|_{L^{p}(J, X)}\right] \\
& +\left(\frac{M \sum_{i=1}^{k} d_{i}\left(t_{i}-t_{i-1}\right)^{\alpha-1}}{\Gamma(\alpha)}+M L b^{1-\alpha} r^{-\alpha}\right) e^{r t}\|x\|_{r} .
\end{aligned}
$$

Hence, we get

$$
\begin{aligned}
& \sup _{t \in\left(t_{k}, t_{k+1}\right]}\left(t-t_{k}\right)^{1-\alpha}\|(\digamma x)(t)\|_{X} e^{-r t} \\
& \leq \omega+\left(\frac{M d_{i} k\left(t_{k}-t_{k-1}\right)^{\alpha-1}}{\Gamma(\alpha)}+M L b^{1-\alpha} r^{-\alpha}\right)\|x\|_{r} \leq R .
\end{aligned}
$$

From the above arguments, we know

$$
\|\digamma x\|_{r}=\max \left\{\sup _{t \in\left(t_{k}, t_{k+1}\right]}\left(t-t_{k}\right)^{1-\alpha}\|(\digamma x)(t)\|_{X} e^{-r t}: k=0,1, \ldots, m\right\} \leq R,
$$

which means that $\digamma\left(B_{r}(R)\right) \subseteq B_{r}(R)$.

Step 2. We show that $\digamma$ is a contraction operator on $B_{r}(R)$.

For any $x, y \in P C_{1-\alpha}(J, X)$, if $t \in\left[0, t_{1}\right]$, then, from formula $(3.1)$, we get

$$
\begin{aligned}
t^{1-\alpha} \|(\digamma x)(t) & -(\digamma y)(t) \|_{X} \leq t^{1-\alpha} \int_{0}^{t}(t-s)^{\alpha-1} \\
& \times\left\|T_{\alpha}(t-s)[f(s, x(s))-f(s, y(s))]\right\| d s
\end{aligned}
$$




$$
\leq \frac{M L t^{1-\alpha}}{\Gamma(\alpha)}\|x-y\|_{r} \int_{0}^{t}(t-s)^{\alpha-1} e^{r s} d s \leq M L b^{1-\alpha} e^{r t} r^{-\alpha}\|x-y\|_{r},
$$

which implies

$$
\sup _{t \in\left[0, t_{1}\right]} t^{1-\alpha}\|(\digamma x)(t)-(\digamma y)(t)\|_{X} e^{-r t} \leq M L b^{1-\alpha} r^{-\alpha}\|x-y\|_{r} \leq \frac{1}{2}\|x-y\|_{r} .
$$

For any $x, y \in P C_{1-\alpha}(J, X)$, if $t \in\left(t_{k}, t_{k+1}\right](k=1, \ldots, m)$. From $H(1)$ and $H(2)$, we get

$$
\begin{aligned}
\left(t-t_{k}\right)^{1-\alpha} \| & (\digamma x)(t)-(\digamma y)(t) \|_{X} \\
\leq & \left(t-t_{k}\right)^{1-\alpha} \| \sum_{i=1}^{k} T_{\alpha}\left(t-t_{i}\right)\left(t-t_{i}\right)^{\alpha-1} \\
& \times\left[G_{i}\left(t_{i}^{-}, x\left(t_{i}^{-}\right)\right)-G_{i}\left(t_{i}^{-}, y\left(t_{i}^{-}\right)\right) \|+\left(t-t_{k}\right)^{1-\alpha}\right. \\
& \times \int_{0}^{t}(t-s)^{\alpha-1}\left\|T_{\alpha}(t-s)[f(s, x(s))-f(s, y(s))]\right\| d s \\
\leq & \left(\frac{M \sum_{i=1}^{k} d_{i}\left(t_{i}-t_{i-1}\right)^{\alpha-1}}{\Gamma(\alpha)}+M L b^{1-\alpha} r^{-\alpha}\right) e^{r t}\|x-y\|_{r} .
\end{aligned}
$$

Then, one can get

$$
\begin{aligned}
& \sup _{t \in\left(t_{k}, t_{k+1}\right]}\left(t-t_{k}\right)^{1-\alpha}\|(\digamma x)(t)-(\digamma y)(t)\|_{X} e^{-r t} \\
\leq & \left(\frac{M \sum_{i=1}^{k} d_{i}\left(t_{i}-t_{i-1}\right)^{\alpha-1}}{\Gamma(\alpha)}+M L b^{1-\alpha} r^{-\alpha}\right)\|x-y\|_{r} \leq \frac{1}{2}\|x-y\|_{r} .
\end{aligned}
$$

Therefore, we obtain

$$
\begin{aligned}
& \|(\digamma x)-(\digamma y)\|_{r} \\
& =\max \left\{\sup _{t \in\left(t_{k}, t_{k+1}\right]}\left(t-t_{k}\right)^{1-\alpha}\|(\digamma x)(t)-(\digamma y)(t)\|_{X} e^{-r t}: k=0,1, \ldots, m\right\} \\
& \leq \frac{1}{2}\|x-y\|_{r}
\end{aligned}
$$

Therefore, $\digamma$ is a contradiction operator. According to Banach's fixed point theorem, we can deduce that problem (1.1) has a unique solution on $J$. The proof is completed. 
Remark 3.2. By a similar technique as applied in Theorem 3.1, we can also derive the existence and uniqueness results for impulsive RiemannLiouville fractional neutral evolution equations in the literature [27] under much weaker conditions.

4. Approximate controllability results. In this section, we research the approximate controllability results of the impulsive fractional evolution differential systems with Riemann-Liouville fractional derivatives.

Let us denote the Nemytskil operator corresponding to the nonlinear function $f$ by:

$$
\Phi_{f}: P C_{1-\alpha}(J, X) \longrightarrow L^{p}(J, X), \quad \Phi_{f}(x)(t)=f(t, x(t)) .
$$

Define the bounded and linear operator $\mathcal{G}: L^{p}(J, X) \rightarrow X$ as

$$
\mathcal{G} h=\int_{0}^{b}(b-s)^{\alpha-1} T_{\alpha}(b-s) h(s) d s, \quad h(\cdot) \in L^{p}(J, X) .
$$

By Definition 2.6, we easily know that if, for any $x_{0} \in X$ and $u(\cdot) \in V, \overline{K_{b}(f)}=X$, then system (1.1) is approximately controllable on $J$. Equivalently, if for every desired final state $\zeta \in X$ and any $\epsilon>0$, there exists a control function $u_{\epsilon}(\cdot) \in V$, such that the mild solution of system (1.1) satisfies

$$
\begin{array}{r}
\| \zeta-b^{\alpha-1} T_{\alpha}(b) x_{0}-\sum_{k=1}^{m}\left(b-t_{k}\right)^{\alpha-1} T_{\alpha}\left(b-t_{k}\right) G_{k}\left(t_{k}^{-}, x_{\varepsilon}\left(t_{k}^{-}\right)\right)-\mathcal{G} \Phi_{f}\left(x_{\varepsilon}\right) \\
-\mathcal{G B} B u_{\varepsilon} \|_{X}<\epsilon,
\end{array}
$$

where $x_{\epsilon}(t)=x\left(t ; u_{\varepsilon}\right), t \in[0, b]$. Then system (1.1) is approximately controllable on $J$.

In order to discuss the approximate controllability of system (1.1), we need:

Lemma 4.1. If the inequality $D^{*}=\max \left\{\left[M k d_{i}\left(t_{k}-t_{k-1}\right)^{\alpha-1} E_{\alpha}(M L b)\right] /\right.$ $\Gamma(\alpha): i=1, \ldots, k ; k=1, \ldots, m+1\}<1$ holds and the conditions 
$H(1)-H(2)$ are satisfied, then any mild solutions of system (1.1) satisfy the following inequalities:

$$
\begin{aligned}
& \left\|x_{1}-x_{2}\right\|_{P C_{1-\alpha}} \\
\leq & \left(1-D^{*}\right) E_{\alpha}(M L b) \frac{M b^{1-1 / p}}{\Gamma(\alpha)}\left(\frac{p-1}{p \alpha-1}\right)^{1-1 / p}\left\|B u_{1}-B u_{2}\right\|_{L^{p}(J, X)},
\end{aligned}
$$

where $x_{j}(j=1,2)$ are mild solutions of system (1.1) corresponding to $u_{j} \in V$, and $E_{\alpha}$ is the Mittag-Leffler function defined by

$$
E_{\alpha}(z)=\sum_{k=0}^{\infty} \frac{z^{k}}{\Gamma(k \alpha+1)} .
$$

Proof. Suppose that $x_{j}(j=1,2)$ are mild solutions of system (1.1) with respect to $u_{j} \in V$ on $P C_{1-\alpha}(J, X)$. Then

$$
\begin{aligned}
x_{j}(t)= & t^{\alpha-1} T_{\alpha}(t) x_{0}+\sum_{k=1}^{m}\left(t-t_{k}\right)^{\alpha-1} T_{\alpha}\left(t-t_{k}\right) G_{k}\left(t_{k}^{-}, x_{j}\left(t_{k}^{-}\right)\right) \\
& +\int_{0}^{t}(t-s)^{\alpha-1} T_{\alpha}(t-s)\left[B u_{j}(s)+f\left(s, x_{j}(s)\right)\right] d s .
\end{aligned}
$$

For any $x_{1}, x_{2} \in P C_{1-\alpha}(J, X), t \in\left(t_{k}, t_{k+1}\right]$, we obtain that

$$
\begin{aligned}
\left(t-t_{k}\right)^{1-\alpha} \| & x_{2}(t)-x_{1}(t) \|_{X} \\
\leq & \left(t-t_{k}\right)^{1-\alpha} \sum_{i=1}^{k}\left(t-t_{i}\right)^{\alpha-1}\left\|T_{\alpha}\left(t-t_{i}\right)\right\| \\
& \times\left\|G_{i}\left(t_{i}^{-}, x_{2}\left(t_{i}^{-}\right)\right)-G_{i}\left(t_{i}^{-}, x_{1}\left(t_{i}^{-}\right)\right)\right\| \\
& +\left(t-t_{k}\right)^{1-\alpha} \int_{0}^{t}(t-s)^{\alpha-1}\left\|T_{\alpha}(t-s)\left(B u_{2}(s)-B u_{1}(s)\right)\right\| d s \\
& +\left(t-t_{k}\right)^{1-\alpha} \int_{0}^{t}(t-s)^{\alpha-1} \| T_{\alpha}(t-s)\left(f\left(s, x_{2}(s)\right)\right. \\
& \\
\leq & \frac{M}{\Gamma(\alpha)} \sum_{i=1}^{k} d_{i}\left(t_{i}-t_{i-1}\right)^{\alpha-1}\left(t_{i}-t_{i-1}\right)^{1-\alpha}\left\|x_{2}\left(t_{i}^{-}\right)-x_{1}\left(t_{i}^{-}\right)\right\|_{X}
\end{aligned}
$$




$$
\begin{aligned}
& +\frac{M b^{1-1 / p}}{\Gamma(\alpha)}\left(\frac{p-1}{p \alpha-1}\right)^{1-1 / p}\left\|B u_{2}-B u_{1}\right\|_{L^{p}(J, X)} \\
& +\frac{M L b^{1-\alpha}}{\Gamma(\alpha)} \int_{0}^{t}(t-s)^{\alpha-1}\left(s-t_{k}\right)^{1-\alpha}\left\|x_{2}(s)-x_{1}(s)\right\|_{X} d s \\
& \leq \frac{M \sum_{i=1}^{k} d_{i}\left(t_{i}-t_{i-1}\right)^{\alpha-1}}{\Gamma(\alpha)}\left\|x_{2}-x_{1}\right\|_{P C_{1-\alpha}} \\
& +\frac{M b^{1-1 / p}}{\Gamma(\alpha)}\left(\frac{p-1}{p \alpha-1}\right)^{1-1 / p}\left\|B u_{2}-B u_{1}\right\|_{L^{p}(J, X)} \\
& +\frac{M L b^{1-\alpha}}{\Gamma(\alpha)} \int_{0}^{t}(t-s)^{\alpha-1}\left(s-t_{k}\right)^{1-\alpha}\left\|x_{2}(s)-x_{1}(s)\right\|_{X} d s .
\end{aligned}
$$

From the above inequality (4.2), it follows from a corollary of Ye et al. [39] that

$$
\begin{aligned}
\left(t-t_{k}\right)^{1-\alpha} \| & x_{2}(t)-x_{1}(t) \|_{X} \\
\leq & {\left[\frac{M \sum_{i=1}^{k} d_{i}\left(t_{i}-t_{i-1}\right)^{\alpha-1}}{\Gamma(\alpha)}\left\|x_{2}-x_{1}\right\|_{P C_{1-\alpha}}\right.} \\
& +\frac{M b^{1-1 / p}}{\Gamma(\alpha)}\left(\frac{p-1}{p \alpha-1}\right)^{1-1 / p} \\
& \left.\quad \times\left\|B u_{2}-B u_{1}\right\|_{L^{p}(J, X)}\right] E_{\alpha}\left(M L b^{1-\alpha} t^{\alpha}\right) .
\end{aligned}
$$

Let $D^{*}=\max \left\{\left[M \sum_{i=1}^{k} d_{i}\left(t_{i}-t_{i-1}\right)^{\alpha-1} E_{\alpha}(M L b)\right] / \Gamma(\alpha): k=\right.$ $0,1, \ldots, m\}$. Therefore, we obtain

$$
\begin{aligned}
& \left\|x_{2}-x_{1}\right\|_{P C_{1-\alpha}} \\
\leq & \left(1-D^{*}\right) E_{\alpha}(M L b) \frac{M b^{1-1 / p}}{\Gamma(\alpha)}\left(\frac{p-1}{p \alpha-1}\right)^{1-1 / p}\left\|B u_{2}-B u_{1}\right\|_{L^{p}(J, X)} .
\end{aligned}
$$

This completes the proof for the Lemma.

In what follows, to discuss the approximate controllability of system (1.1), we assume:

$H(3)$ : For any $\varepsilon>0$ and $\varphi \in L^{p}(J, X)$, there exists a control $u \in L^{p}(J, U)$ such that

$$
\|\mathcal{G} \varphi-\mathcal{G} B u\|_{X}<\varepsilon
$$




$$
\|B u\|_{L^{p}(J, X)}<N\|\varphi\|_{L^{p}(J, X)},
$$

where $N$ is a constant which is independent of $\varphi \in L^{p}(J, X)$, and

$$
N\left(1-D^{*}\right) E_{\alpha}(M L b) \frac{M b^{1-1 / p}}{\Gamma(\alpha)}\left(\frac{p-1}{p \alpha-1}\right)^{1-1 / p}<1 .
$$

Theorem 4.2. Suppose that the hypotheses of Lemma 4.1 and $H(3)$ hold. Then system (1.1) is approximately controllable on $J$, if the $C_{0}$-semigroup $T(t)$ generated by $A$ is differentiable on the Banach space $X$.

Proof. Since the domain $D(A)$ of operator $A$ is dense in $X$, it is sufficient to show that $D(A) \subset K_{b}(f)$, i.e., for any $\epsilon>0$ and $\eta \in D(A)$, there exists a $u_{\epsilon} \in V$, such that

$$
\begin{array}{r}
\| \eta-b^{\alpha-1} T_{\alpha}(b) x_{0}-\sum_{k=1}^{m}\left(b-t_{k}\right)^{\alpha-1} T_{\alpha}\left(b-t_{k}\right) G_{k}\left(t_{k}^{-}, x_{\varepsilon}\left(t_{k}^{-}\right)\right) \\
-\mathcal{G} \Phi_{f}\left(x_{\varepsilon}\right)-\mathcal{G} B u_{\varepsilon} \|_{X}<\epsilon,
\end{array}
$$

where $x_{\epsilon}(t)=x\left(t ; u_{\varepsilon}\right)$ and $t \in[0, b]$.

Firstly, for any $x_{0} \in X$, due to the differentiability of the $C_{0^{-}}$ semigroup $T(t)(t>0)$, we know that $b^{\alpha-1} T_{\alpha}(b) x_{0} \in D(A)$. Therefore, for any given $\eta \in D(A)$, it can be seen that there exists a function $\varphi \in L^{p}(J, X)$ such that

$$
\mathcal{G} \varphi=\eta-b^{\alpha-1} T_{\alpha}(b) x_{0},
$$

for example,

$$
\varphi(t)=\frac{[\Gamma(\alpha)]^{2}(b-t)^{1-\alpha}}{b}\left[\begin{array}{c}
\left.T_{\alpha}(b-t)-2 t \frac{d T_{\alpha}(b-t)}{d t}\right] \\
{\left[\eta-b^{\alpha-1} T_{\alpha}(b) x_{0}\right], \quad t \in(0, b) .}
\end{array}\right.
$$

Similarly, for every $x_{i} \in X, i=1,2, \ldots$, we can also get a function $\varphi_{i} \in$ $L^{p}(J, X)$, such that $\mathcal{G} \varphi_{i}=\sum_{k=1}^{m}\left(b-t_{k}\right)^{\alpha-1} T_{\alpha}\left(b-t_{k}\right) G_{k}\left(t_{k}^{-}, x_{i}\left(t_{k}^{-}\right)\right)$.

Next, we show that one can get a control function $u_{\epsilon} \in V$ such that the inequality (4.6) holds. In fact, for any given $\epsilon>0$ and $u_{1} \in V$, 
from $H(3)$, there exists a $u_{2} \in V$, such that

$$
\begin{array}{r}
\| \eta-b^{\alpha-1} T_{\alpha}(b) x_{0}-\sum_{k=1}^{m}\left(b-t_{k}\right)^{\alpha-1} T_{\alpha}\left(b-t_{k}\right) G_{k}\left(t_{k}^{-}, x_{1}\left(t_{k}^{-}\right)\right) \\
-\mathcal{G} \Phi_{f}\left(x_{1}\right)-\mathcal{G B} u_{2} \|_{X}<\frac{\epsilon}{2^{2}},
\end{array}
$$

where $x_{1}(t)=x\left(t ; u_{1}\right), 0 \leq t \leq b$. Denote $x_{2}(t)=x\left(t ; u_{2}\right), 0 \leq t \leq b$. By the hypotheses $H(3)$ again, there exists $w_{2} \in V$ such that

$$
\begin{gathered}
\| \mathcal{G B} w_{2}-\left[\mathcal{G} \Phi_{f}\left(x_{2}\right)+\sum_{k=1}^{m}\left(b-t_{k}\right)^{\alpha-1} T_{\alpha}\left(b-t_{k}\right) G_{k}\left(t_{k}^{-}, x_{2}\left(t_{k}^{-}\right)\right)\right. \\
\left.\mathcal{G} \Phi_{f}\left(x_{1}\right)-\sum_{k=1}^{m}\left(b-t_{k}\right)^{\alpha-1} T_{\alpha}\left(b-t_{k}\right) G_{k}\left(t_{k}^{-}, x_{1}\left(t_{k}^{-}\right)\right)\right] \|_{X} \leq \frac{\epsilon}{2^{3}} .
\end{gathered}
$$

and

$$
\begin{aligned}
\left\|B w_{2}\right\|_{L^{p}(J, X) \leq} & N\left\|\Phi_{f}\left(x_{2}\right)(\cdot)-\Phi_{f}\left(x_{1}\right)(\cdot)\right\|_{X} \\
\leq & N L\left(t-t_{k}\right)^{1-\alpha}\left\|x_{2}(\cdot)-x_{1}(\cdot)\right\|_{X} \\
\leq & N\left(1-D^{*}\right) E_{\alpha}(M L b) \frac{M b^{1-1 / p}}{\Gamma(\alpha)}\left(\frac{p-1}{p \alpha-1}\right)^{1-1 / p} \\
& \times\left\|B u_{1}-B u_{2}\right\|_{L^{p}(J, X)} .
\end{aligned}
$$

Now, we define

$$
u_{3}(t)=u_{2}(t)-w_{2}(t) \quad u_{3} \in V
$$

and it follows that

$$
\begin{gathered}
\| \eta-b^{\alpha-1} T_{\alpha}(b) x_{0}-\sum_{k=1}^{m}\left(b-t_{k}\right)^{\alpha-1} T_{\alpha}\left(b-t_{k}\right) G_{k}\left(t_{k}^{-}, x_{2}\left(t_{k}^{-}\right)\right) \\
\quad-\mathcal{G} \Phi_{f}\left(x_{2}\right)-\mathcal{G} B u_{3} \|_{X} \\
\leq \| \eta-b^{\alpha-1} T_{\alpha}(b) x_{0}-\sum_{k=1}^{m}\left(b-t_{k}\right)^{\alpha-1} T_{\alpha}\left(b-t_{k}\right) G_{k}\left(t_{k}^{-}, x_{1}\left(t_{k}^{-}\right)\right) \\
-\mathcal{G} \Phi_{f}\left(x_{1}\right)-\mathcal{G B} u_{2} \|_{X}
\end{gathered}
$$




$$
\begin{aligned}
& +\| \mathcal{G B} w_{2}-\left[\mathcal{G} \Phi_{f}\left(x_{2}\right)+\sum_{k=1}^{m}\left(b-t_{k}\right)^{\alpha-1} T_{\alpha}\left(b-t_{k}\right) G_{k}\left(t_{k}^{-}, x_{2}\left(t_{k}^{-}\right)\right)\right. \\
& \left.-\mathcal{G} \Phi_{f}\left(x_{1}\right)-\sum_{k=1}^{m}\left(b-t_{k}\right)^{\alpha-1} T_{\alpha}\left(b-t_{k}\right) G_{k}\left(t_{k}^{-}, x_{1}\left(t_{k}^{-}\right)\right)\right] \|_{X} \\
& \leq\left(\frac{1}{2^{2}}+\frac{1}{2^{3}}\right) \epsilon .
\end{aligned}
$$

By inductions, we can get the sequence $\left\{u_{n}(\cdot)\right\} \subset V$ from which it follows that

$$
\begin{array}{r}
\| \eta-b^{\alpha-1} T_{\alpha}(b) x_{0}-\sum_{k=1}^{m}\left(b-t_{k}\right)^{\alpha-1} T_{\alpha}\left(b-t_{k}\right) G_{k}\left(t_{k}^{-}, x_{n}\left(t_{k}^{-}\right)\right) \\
-\mathcal{G} \Phi_{f}\left(x_{n}\right)-\mathcal{G} B u_{n+1} \|_{X}<\left(\frac{1}{2^{2}}+\cdots+\frac{1}{2^{n}}\right) \epsilon
\end{array}
$$

where $x_{n}(\cdot)=x\left(\cdot ; u_{n}\right), 0 \leq t \leq b$, and

$$
\begin{aligned}
\left\|B u_{n+1}-B u_{n}\right\|_{L^{p}(J, X)} \leq & \left(1-D^{*}\right) E_{\alpha}(M L b) \frac{M b^{1-1 / p}}{\Gamma(\alpha)}\left(\frac{p-1}{p \alpha-1}\right)^{1-1 / p} \\
& \times\left\|B u_{n}-B u_{n-1}\right\|_{L^{p}(J, X)} .
\end{aligned}
$$

From (4.5), we know that the sequence $\left\{B u_{n}: n=1,2, \ldots\right\}$ is a Cauchy sequence on the Banach space $L^{p}(J, X)$. Therefore, there exists a sequence $\psi(\cdot) \in L^{p}(J, X)$, such that

$$
\lim _{n \rightarrow \infty} B u_{n}(\cdot)=\psi(\cdot) \quad \text { in } L^{p}(J, X) .
$$

Then, for any $\epsilon>0$, there exists a positive integer number $N$, such that

$$
\left\|\mathcal{G B} u_{N+1}-\mathcal{G} B u_{N}\right\|_{X}<\frac{\epsilon}{2} .
$$

Therefore, we have

$$
\begin{array}{r}
\| \eta-b^{\alpha-1} T_{\alpha}(b) x_{0}-\sum_{k=1}^{m}\left(b-t_{k}\right)^{\alpha-1} T_{\alpha}\left(b-t_{k}\right) G_{k}\left(t_{k}^{-}, x_{N}\left(t_{k}^{-}\right)\right) \\
-\mathcal{G} \Phi_{f}\left(x_{N}\right)-\mathcal{G B} u_{N} \|_{X}
\end{array}
$$




$$
\begin{aligned}
\leq & \| \eta-b^{\alpha-1} T_{\alpha}(b) x_{0}-\sum_{k=1}^{m}\left(b-t_{k}\right)^{\alpha-1} T_{\alpha}\left(b-t_{k}\right) G_{k}\left(t_{k}^{-}, x_{N}\left(t_{k}^{-}\right)\right) \\
& \quad-\mathcal{G} \Phi_{f}\left(x_{N}\right)-\mathcal{G} B u_{N+1} \|_{X} \\
& +\left\|\mathcal{G B} u_{N+1}-\mathcal{G B} u_{N}\right\|_{X} \leq\left(\frac{1}{2^{2}}+\cdots+\frac{1}{2^{n}}\right) \epsilon+\frac{\epsilon}{2}<\epsilon
\end{aligned}
$$

This proves the approximate controllability of system (1.1).

5. An example. Consider the following initial-boundary value problem of fractional parabolic control system with Riemann-Liouville fractional derivatives:

$$
\begin{cases}D_{t}^{4 / 5} x(t, y)=\frac{\partial^{2}}{\partial y^{2}} x(t, y)+f(t, x(t))+B u(t), & t \in J=[0,1] \backslash\left\{\frac{1}{2}\right\}, \\ \Delta I_{t}^{1 / 5} x\left(\frac{1}{2}, y\right)=\frac{|x(y)|}{2+|x(y)|}, & y \in[0, \pi], \\ x(t, 0)=x(t, \pi)=0, & y \in[0, \pi] \\ \left.I_{t}^{1 / 5} x(t, y)\right|_{t=0}=x_{0}(y), & t \in J=[0,1], \\ & t \in[0,1], \\ & y \in[0, \pi] .\end{cases}
$$

Take $X=U=L^{2}([0, \pi])$, and the operator $A: D(A) \subset X \rightarrow X$ is defined by

$$
A x=x^{\prime \prime}
$$

$$
\begin{aligned}
& D(A)=\left\{x \in X: x, x^{\prime}\right. \text { are absolutely continuous, } \\
& \left.\qquad x^{\prime \prime} \in X, x(0)=x(\pi)=0\right\} .
\end{aligned}
$$

Then, $A$ can be written as

$$
A x=-\sum_{n=1}^{\infty} n^{2}\left(x, x_{n}\right) x_{n}, \quad x \in D(A)
$$

where $x_{n}(x)=\sqrt{2 / \pi} \sin n y(n=1,2, \ldots)$ is an orthonormal basis of $X$. It is well known that $A$ is the infinitesimal generator of a differentiable 
semigroup $T(t)(t>0)$ in $X$ given by

$$
T(t) x=\sum_{n=1}^{\infty} \exp ^{-n^{2} t}\left(x, x_{n}\right) x_{n}, \quad x \in X,
$$

and

$$
\|T(t)\| \leq e^{-1}<1=M
$$

For every $u(\cdot) \in V=L^{2}(J, U)$, we have

$$
u(t)=\sum_{n=1}^{\infty} u_{n}(t) x_{n}, \quad u_{n}(t)=\left\langle u(t), x_{n}\right\rangle .
$$

Define the operator $B$ as

$$
B u(t)=\sum_{n=1}^{\infty} \bar{u}_{n}(t) x_{n}
$$

where

$$
\bar{u}_{n}(t)=\left\{\begin{array}{cl}
0, & 0 \leq t<1-1 / n^{2}, \\
u_{n}(t), & 1-1 / n^{2} \leq t \leq 1,
\end{array} \quad n=1,2, \ldots\right.
$$

Then, one can easily obtain that $\|B u(\cdot)\| \leq\|u(\cdot)\|$, which implies that $B \in L\left(V, L^{2}(J, X)\right)$.

Firstly, by the definition of the operator $B$, the corresponding linear system of (5.1) is presented as follows:

$$
\left\{\begin{array}{l}
D_{t}^{4 / 5} x_{n}(t)+n^{2} x_{n}(t)=\widehat{u}_{n}(t), \quad 1-1 / n^{2}<t<1 \\
\Delta I_{t}^{1 / 5} x_{n}(1 / 2)=\frac{\left|x_{n}(y)\right|}{2+\left|x_{n}(y)\right|} \\
\left.I_{t}^{1 / 5} x_{n}(t)\right|_{t=0}=x_{0} \in X
\end{array}\right.
$$

Next, we will check that hypothesis $H(4)$ is satisfied. To check this, let us denote

$$
\begin{aligned}
h & =\int_{0}^{1}(1-s)^{-1 / 5} T_{4 / 5}(1-s) g(s) d s \\
& =\sum_{n=1}^{\infty} h_{n} x_{n}, h_{n}=\left\langle h, x_{n}\right\rangle, \quad \text { for every } g(\cdot) \in L^{2}(J, X) .
\end{aligned}
$$


In fact, we can choose $\widetilde{u}_{n}(t)$, which follows from

$$
\widetilde{u}_{n}(t)=\frac{3 n^{2}}{1-e^{-2}} h_{n} e^{-n^{2}(1-t)}, \quad 1-\frac{1}{n^{2}} \leq t \leq 1
$$

and

$$
h_{n}=\int_{1-1 / n^{2}}^{1} \int_{0}^{\infty}(1-t)^{-1 / 5} \theta \xi_{4 / 5}(\theta) e^{-n^{2} \theta(1-t)^{4 / 5}} \widetilde{u}_{n}(t) d \theta d t .
$$

For this, we define

$$
u(t)=\sum_{n=1}^{\infty} u_{n}(t) x_{n}
$$

where

$$
u_{n}(t)=\left\{\begin{array}{cc}
0, & 0 \leq t<1-1 / n^{2}, \\
\widetilde{u}_{n}(t), & 1-1 / n^{2} \leq t \leq 1,
\end{array} \quad n=1,2, \ldots\right.
$$

Therefore, for any given function $g(\cdot) \in L^{2}([0,1], X)$, there exists $u(\cdot) \in V$ such that

$$
\int_{0}^{1}(1-s)^{-1 / 5} T_{4 / 5}(1-s) B u(s) d s=\int_{0}^{1}(1-s)^{-1 / 5} T_{4 / 5}(1-s) g(s) d s,
$$

which implies that condition (4.2) of $H(4)$ is satisfied. Moreover, we can get

$$
\begin{aligned}
\|B u(\cdot)\|^{2} & =\sum_{n=1}^{\infty} \int_{1-1 / n^{2}}^{1}\left|\widetilde{u}_{n}(t)\right|^{2} d t=\left(1-e^{-2}\right)^{-1} \sum_{n=1}^{\infty} 3 n^{2} h_{n}^{2} \\
& =3\left(1-e^{-2}\right)^{-1} \sum_{n=1}^{\infty}\left(1-e^{-2 n^{2}}\right) \int_{0}^{1}\left|g_{n}(t)\right|^{2} d t \\
& \leq\left(1-e^{-2}\right)^{-1}|g(\cdot)|^{2}
\end{aligned}
$$

Hence, it can be seen that if condition $H(4)$ is satisfied, then system (5.1) is approximately controllable on $J$, if

$$
\frac{3\left(1-E_{4 / 5}(L) / 2^{\alpha} \Gamma(4 / 5)\right) E_{4 / 5}(L)}{\left(1-e^{-2}\right) \Gamma(4 / 5)}<1,
$$

is satisfied. 


\section{REFERENCES}

1. N. Abada, M. Benchohra and H. Hammouche, Existence and controllability results for nondensely defined impulsive semilinear functional differential inclusions, J. Diff. Equat. 246 (2009), 3834-3863.

2. D. Baleanu and A.K. Golmankhaneh, On electromagnetic field in fractional space, Nonlin. Anal.: RWA 11 (2010), 288-292.

3. A.E. Bashirov and N.I. Mahmudov, On concepts of controllability for deterministic and stochastic systems, SIAM J. Cont. Optim. 37 (1999), 1808-1821.

4. M. Benchohra and A. Ouahab, Controllability results for functional semilinear differential inclusions in Frechet spaces, Nonlin. Anal.: TMA. 61 (2005), $405-423$.

5. Y.K. Chang and D.N. Chalishajar, Controllability of mixed VolterraFredholm-type integro-differential inclusions in Banach spaces, J. Franklin Inst. 345 (2008), 499-507.

6. Y.K. Chang, J.J. Nieto and W.S. Li, Controllability of semilinear differential systems with nonlocal initial conditions in Banach spaces, J. Optim. Theor. Appl. 142 (2009), 267-273.

7. Y.K. Chang, J.J. Nieto and Z.H. Zhao, Existence results for a nondenselydefined impulsive neutral differential equation with state-dependent delay, Nonlin. Anal. 4 (2010), 593-599.

8. X. Fu, Controllability of non-densely defined functional differential systems in abstract space, Appl. Math. Lett. 19 (2006), 369-377.

9. L. Gorniewicz, S.K. Ntouyas and D. O'Regan, Controllability of semilinear differential equations and inclusions via semigroup theory in Banach spaces, Rep. Math. Phys. 56 (2005), 437-470.

10. H. Henríquez and M. Pierri, Approximate controllability of systems determined by almost sectorial operators, Z. Anal. Anw. 32 (2013), 199-214.

11. E. Hernández, D. O'Regan and E. Balachandran, On recent developments in the theory of abstract differential equations with fractional derivatives, Nonlin. Anal. 73 (2010), 3462-3471.

12. N. Heymans and I. Podlubny, Physical interpretation of initial conditions for fractional differential equations with Riemann-Liouville fractional derivatives, Rheol. Acta. 45 (2006), 765-771.

13. R.E. Kalman, Controllablity of linear dynamical systems, Contrib. Diff. Eq. 1 (1963), 190-213.

14. A.A. Kilbas, H.M. Srivastava and J.J. Trujillo, Theory and applications of fractional differential equations, in North-Holland Math. Stud. 204, Elservier Science B.V., Amsterdam, 2006.

15. J. Klamka, Constrained approximate controllability, IEEE Trans. Autom. Contr. 45 (2000), 1745-1749.

16. S. Kumar and N. Sukavanam, Approximate controllability of fractional order semilinear systems with bounded delay, J. Diff. Eq. 252 (2012), 6163-6174. 
17. V. Lakshmikantham, S. Leela and J. Vasundhara Devi, Theory of fractional dynamic systems, Cambridge Academic Publishers, Cambridge, 2009.

18. M.L. Li, Controllability of nonlinear third-order dispersion inclusions with infinite delay, Electron J. Diff. Eq. 170 (2013), 1-10.

19. W.S. Li, Y.K. Chang and J.J. Nieto, Solvability of impulsive neutral evolution differential inclusions with state-dependent delay, Math. Comp. Model. 49 (2009), 1920-1927.

20. J.T. Liang, Y.L. Liu and Z.H. Liu, A class of BVPS for first order impulsive integrodifferential equations, Appl. Math. Comp. 218 (2011), 3667-3672.

21. Z.H. Liu and M.J. Bin, Approximate controllability for impulsive RiemannLiouville fractional differential inclusions, Abs. Appl. Anal. 2013, Article ID 639492, 17 pages.

22. Z.H. Liu and X.W. Li, Approximate controllability for a class of hemivariational inequalities, Nonlin. Anal. Real World 22 (2015), 581-591.

23. Existence and uniqueness of solutions for the nonlinear impulsive fractional differential equations, Comm. Nonlin. Sci. Num. Sim. 18 (2013), 13621373.

24. On the controllability of impulsive fractional evolution inclusions in Banach spaces, J. Optim. Theor. Appl. 156 (2013), 167-182.

25. Z.H. Liu, X.W. Li and J.H. Sun, Controllability of nonlinear fractional impulsive evolution systems, J. Int. Eq. Appl. 25 (2013), 395-406.

26. Z.H. Liu and J.T. Liang, A class of boundary value problems for first-order impulsive integro-differential equations with deviating arguments, J. Comp. Appl. Math. 237 (2013), 477-486.

27. X.H. Liu, Z.H. Liu and M.J. Bin, Approximate controllability of impulsive fractional neutral evolution equations with Riemann-Liouville fractional derivatives, J. Comp. Anal. Appl. 17 (2014), 468-485.

28. Z.H. Liu and J.H. Sun, Nonlinear boundary value problems of fractional functional integro-differential equations, Comp. Math. Appl. 64 (2012), 3228-3234.

29. N.I. Mahmudov, Approximate controllability of fractional Sobolev-type evolution equations in Banach spaces, Abst. Appl. Anal. 2013, Art. ID 502839, 1-9.

30. X. Pan, X.W. Li and J. Zhao, Solvability and optimal controls of semilinear Riemann-Liouville fractional differential equations, Abst. Appl. Anal. 2014, Article ID 216919, 11 pages, http://dx.doi.org/10.1155/2014/216919.

31. A. Pazy, Semigroups of linear operators and applications to partial differential equations, Springer-Verlag, New York, 1983.

32. I. Podlubny, Fractional differential equations, Academic Press, San Diego, 1999.

33. K. Rykaczewski, Approximate controllability of differential of fractional inclutions in Hilbert spaces, Nonlin. Anal. 75 (2012), 2701-2712.

34. R. Sakthivel, E.R. Anandhi and S.G. Lee, Approximate controllability of impulsive differential inclusions with nonlocal conditions, Dynam. Syst. Appl. 18 (2009), 637-654. 
35. R. Sakthivel, Y. Ren and N. I. Mahmudov, On the approximate controllability of semilinear fractional differential systems, Comp. Math. Appl. 62 (2011), 1451-1459.

36. N. Sukavanam and Surendra Kumar, Approximate controllability of fractional order semilinear delay systems, J. Optim. Theor. Appl. 151 (2011), 373-384.

37. R. Triggiani, A note on the lack of exact controllability for mild solutions in Banach spaces, SIAM J. Cont. Optim. 15 (1977), 407-411.

38. J.R. Wang, M. Fečkan and Y. Zhou, On the new concept of solutions and existence results for impulsive fractional evolution equations, Dynam. PDE 8 (2011), 345-361.

39. H.P. Ye, J.M. Gao and Y.S. Ding, A generalized Gronwall inequality and its application to a fractional differential equation, J. Math. Anal. Appl. 328 (2007), 1075-1081.

40. H.X. Zhou, Approximate controllability for a class of semilinear abstract equations, SIMA J. Cont. Optim. 22 (1983), 405-422.

41. Y. Zhou and F. Jiao, Existence of mild solutions for fractional neutral evolution equations, Comp. Math. Appl. 59 (2010), 1063-1077.

Guangxi Key Laboratory of Universities Optimization Control and Engineering Calculation, and College of Sciences, Guangxi University for Nationalities, Nanning, Guangxi 530006, P.R. China

\section{Email address: zhhliu@hotmail.com}

College of Sciences, Guangxi University for Nationalities, Nanning, Guangxi 530006, P.R. China

Email address: bmj1999@163.com 